\title{
Finite Element Modeling of Atlantoaxial Joint with the Vertebral Artery Based on CT Data
}

\author{
Shaoyin Duan ${ }^{1, \text { a }}$, Li Zhang ${ }^{1}$, Changhua Lin ${ }^{1}$ and Hua Zhong ${ }^{1}$ \\ ${ }^{1}$ Department of Medical Imaging, Zhongshan Hospital of Xiamen University, Xiamen 361004, China \\ axmdsy@xmzsh.com
}

Keywords: Atlantoaxial joint; Vertebral artery; CT data; Finite element modeling

\begin{abstract}
Based on the data of atlantoaxial joint (AAJ) by contrast-enhanced CT scan, finite element modeling (FEM) of AAJ with vertebral artery (VA) was constituted and its feasibility and possible application were evaluated. CT data of AAJ with VA was selected from PACS in our hospital. The occipital, vertebral body and VA were distinguished and saved them as the STL format, the geometric and finite element model of AAJ with VA were established with adjusting the relevant parameters. Comparison was made between the model and 3D-image, and AAJ functions were simulated. FEM cannot clearly only show or measure the morphology of AAJ and VA, but observe the AAJ rotary motion and have a force analysis. In conclusion, FEM of AAJ with VA is a practical method on the CT data, and it has a high reliability, and can be used for studying the anatomy and biomechanics of AAJ.
\end{abstract}

\section{Introduction}

Atlantoaxial joint (AAJ) is the region of cranio-spinal junction, with the complexity structure and function. It mainly includes the atlantoaxial vertebrae, lower part of medulla, spinal cord of cervical segment, atlantoaxial ligament, vertebral artery (VA) and vertebral venous plexus and so on. As special features of the regional anatomy and functions, their abnormal are mainly in AAJ deformity, vertebral artery variation, local fracture or dislocation of cervical vertebra, it can cause the AAJ instability and the upper cervical spinal cord compression, therefore, great harm to human health, and may lead to cervical paralysis and endanger the life [1,2]. With the development of imaging CT, MRI technology, 3D-imaging and FEM were used to observe the AAJ, VA and their relationship with the surrounding ligaments $[3,4]$. They can provide morphological and functional basis for the study on the anatomy, biomechanics of the AAJ and VA, and improve the level of clinical diagnosis and treatment related to the AAJ and VA diseases.

\section{Materials and Methods}

General Information and Equipment. CT scan data selected from 88 cases CTA scan data of AAJ and VA without rotation and lesion by 3D-imaging display. Spiral CT scanner for multi-slice spiral CT (Multi-detector-row spiral CT, MRCT, including Light Speed16 or Light Speed VCT, GE company, USA) were used, as well as the image processing workstation (Advantage workstation aw 4.2, GE company, USA), Pressure syringe for single or double tube (MCT-plus type PGH. or STELLANT, MEDRAD company, USA) and Contrast agent (Omnipaque, 300 $\mathrm{mgI} / \mathrm{ml}$ ), a total of $1.5 \mathrm{ml} \sim 2 \mathrm{ml} / \mathrm{kg}$, rate of $3 \mathrm{ml} / \mathrm{s} \sim 4 \mathrm{ml} / \mathrm{s}$.

Date Segment. Occipital bone $(\mathrm{C} 0)$, atlas $(\mathrm{C} 1)$, axis $(\mathrm{C} 2)$, third cervical vertebrae (C3) and VA were segmented by using the threshold technique and growth method, which can be used due to their high CT value and data distribution in three-dimensional space. The growth method is a simple and effective mean. First there is a hand selection of target structure region growing seed points, then have a growth with the gray exceeds a certain threshold volume in the three-dimensional space. Before the growth, each image respectively have the Gauss-filter and $3 \times 3$ median-filter to improve the segmentation results, which the bone and joint surface is smooth and clear. In the process of region growing algorithm, there are two criteria: 1 . CT value of the growth point is greater than a specified threshold; 2 . the growth point distance is in a given range, this criterion can ensure the 
algorithm to continue.

Establishment of Geometric Model. Due to the structure complexity of the geometric model of AAJ and VA, the author uses the point surface modeling method. Each vertebral body and VA was distinguished and saved as STL format files using threshold and manual binding technique, and imported the MIMICS software. In MIMICS, AAJ and VA were divided into a plurality of regions according to the structure change of curvature, and the geometric model of AAJ with VA formed using point cloud data.

Establishment of FEM. firstly, mapped mesh of AAJ and VA. For their different structures, the different unit types were used to simulate. Content include: shell elements are used for the cortical bone of vertebral body, average thickness is $0.625 \mathrm{~mm}$; solid element for cancellous bone, as well as the disc and VA. The ligaments are fibrous tissue with tension load, atlantoaxial ligament such as anterior longitudinal ligament, posterior longitudinal ligament, interspinal ligament and interspinous ligament. They were used the linear membrane element with tension property, membrane unit thickness is $0.625 \mathrm{~mm}$. There are 91967 solid unit and 367868 shell / membrane element in the FEM of AAJ and VA.

Relevant Material Parameters. These parameters were form the reference papers [6]. Atlantoaxial bone using isotropic elastic material, cortical bone was the density of $1.83 \mathrm{~g} / \mathrm{cm}$, Young's modulus of $1.2 \mathrm{Gpa}$ and Poisson's ratio of 0.29 . Cancellous bone was the density of $1 \mathrm{~g} / \mathrm{cm}$, Young's modulus of $450 \mathrm{Mpa}$ and Poisson's ratio of 0.29. Nucleus pulposus of intervertebral disc was the short-acting shear modulus $\left(\mathrm{G}_{0}\right)$ of $2 \mathrm{MPa}$, long-term shear modulus $\left(\mathrm{G}_{\infty}\right)$ of $1.4 \mathrm{MPa}$, the bulk modulus of $2.2 \mathrm{GPa}$. Fiber ring was set as a linear elastic material, density of $1.2 \mathrm{~g} / \mathrm{cm}$, Young's modulus of 3.4 Mpa and Poisson's ratio of 0.4. Eendplate was the density of $1.83 \mathrm{~g} / \mathrm{cm}$, Young's modulus of $500 \mathrm{Mpa}$ and Poisson's ratio of 0.29 . Ligament was the density of $1.1 \mathrm{~g} / \mathrm{cm}$, the Poisson's ratio of 0.4 and Young's modulus of anterior longitudinal ligament in $11.4 \mathrm{MPa}$, posterior longitudinal ligament in $9.12 \mathrm{Mpa}$, supraspinous ligament in $8.55 \mathrm{Mpa}$, cruciate ligament in 17.1 $\mathrm{Mpa}$, alar ligament in 11.4 Mpa and joint capsule ligament in $22.8 \mathrm{MPa}$.

\section{Results}

CT data of AAJ with VA meet the requirements of FEM. The bone structures including the occipital bone $(\mathrm{C} 0)$, atlas $(\mathrm{C} 1)$, axis $(\mathrm{C} 2)$, third cervical vertebrae $(\mathrm{C} 3)$ and VA were clearly showed and easily distinguished, but the figments of AJJ were not easy to segment because their structure is small.

FEM of AAJ with VA, which is similar with CT 3D-image. By segmenting the C0, C1, C2, C3 and VA, these structures finally gets clearly display and realize the imaging separate and fusion. FEM of AAJ with VA can be rotated any angle or direction in the three-dimensional space, and measured all of structures.

Modeling method with CT data was simple and not difficult. Compared with CT three-dimensional image, the FEM cannot only clearly show the structure and morphology of AAJ and VA, but can observe the AAJ rotary motion, and have a force analysis by exerting force (Fig. $1)$.
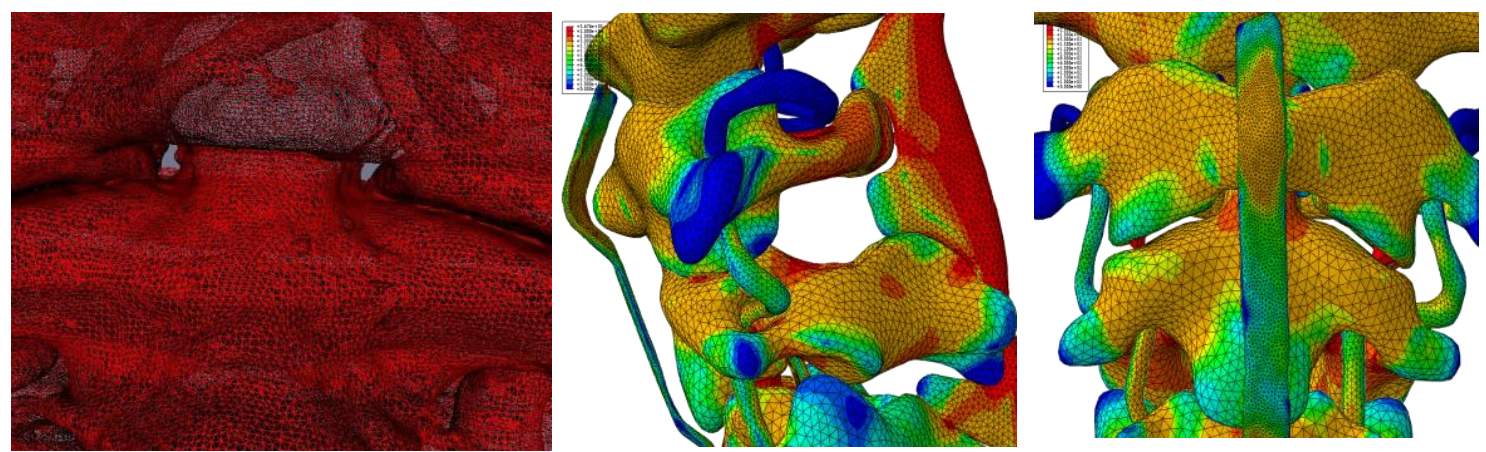

Figure 1. Local image of AAJ after mesh generation (A), The stress distribution cloud pictures of FEM of AAJ with VA on $5 \mathrm{~N}$ loading (B-lateral view, C-frontal view) 


\section{Discussion}

Modeling Process. Used CTA imaging data of head and neck, AAJ and VA can be clearly segmented based on the deformable contour and automatic segmentation. This is the first step to construct 3D geometric model and FEM. In order to improve the modeling speed and accuracy of segmentation, the algorithm should also consider the direct segmentation operation in three-dimensional space. On this basis, the adaptive triangulation algorithm should be used. In the area of complex structure, the more and finer tetrahedral were meshed, then in the area of simple structure or a single physical properties, the less and coarse tetrahedral were generated. Only in this way, the tetrahedral number of FEM can effectively be controlled and adjusted to ensure the FEM with suitable size, which can be used to finish the functional simulation and calculation, and it was close to the actual structure as far as possible. Finally, it is the point to select the parameters of physical properties and material characteristics about AAJ, VA and ligaments [3, 5]. These parameters can be from previous studies and own experiments, which also are the important research contents.

Biomechanics Research. Research methods of biomechanics include animal experiment, physical experiment, cadaver experiment and computer simulation, of which the cadaver experiments were the ideal, their results can be used for the direction and evaluation the effect of surgical operation. However, the cadaver experiment lacks the biological characteristics, and the cost is relatively high. Besides, the cadaver specimens are difficult to get. The computer simulation has a certain advantages with a relatively low costs, it can be repeated by changing the parameters of experiment. Computer simulation can provide the mechanical changes of local structures, and supple the experimental deficiency of cadaveric specimens $[5,6]$. In the theory, FEM technique is applicable to any complex structure and functional simulation, but in practical application, there exist many problems need to solve. The simulation results should be had a theoretical basis and analyzed or compared with the experiments in vitro or vivo, this is a very important steps. FEM of AAJ with VA is a collective body of the AAJ, VA finite element with limited degrees of freedom. Their nodes connected the unit to unit, whose action transfer through nodes. Based on relationship with node, displacement and nodal force, each unit with material properties, boundary conditions and load was set, its stiffness matrix was calculated and had a mathematical expression to observe the biomechanical changes. By changed the related parameters, the biomechanics characteristics of AAJ were achieved, as well as the fluid mechanics of VA.

Accuracy of FEM. influence factors of FEM accuracy include the finite element grid and model material definition. About grid, the hexahedron element with eight nodes was adopted, and strictly controlled the quality of the model unit. Defining the model material is a key part, AAJ related materials data were scarce, because of the late start about the researches. Early model material definition is relatively simple, for example, the model was from the Kleinberger [7], its vertebrae simply defined as rigid material, disc as a elastic material and ligament as a linear elastic material and so on. In fact, there was a certain gap with the actual cervical vertebra. In addition, some parameters were from the lumbar spine, although there are some similar, but the mechanical function, structure characteristics are different. This study based on existing cervical material on the experimental data and the characteristics of cervical structures, there were an appropriate scaling about material parameters of AAJ, our believed that will make the material parameters of AAJ structures more representative. Of course, the material parameters of model proposed by this study, that must not meet the body's physiological standard and still need to constantly enrich, perfect in the future study. This is a continuous process of development, including the verification and supplement of the new physiological parameters.

Modeling Data and Methods. CT data is from our hospital, it is rich and easy to achieve. The data is objective and include the true anatomic structures. Used the CT data, the modeling is simple and model is reliable. Compared another modeling method used CAD data, the method used CT data will greatly simplify the whole of modeling process, reduce the modeling difficulty, and reduce the modeling time. It was easy to grasp for the radiologist to obtain the anatomical structure with high precision, which can provided the conditions and basis for the study of the AAJ and VA 
related diseases. Based on FEM of AAJ with VA, the interactive simulation technology can actually be operated, including the 3D-roaming, 3D-display and 3D-control, which have been many mature experiences, they can be directly used in the functional simulation of AAJ and VA [5, 6]. Interacted with the mouse and the FEM and applied various loads, the surface dislocation of AAJ can be had an accurate calculation and measurement, as well as the biomechanical changes on the AAJ, ligament and VA and so on. The final results were displayed in an intuitive graph. This results might reveal the abnormal anatomy and pathology of AAJ dislocation and VA lumen stenosis, and provide a tool and platform for studying on the diagnosis and treatment of related to AAJ and VA diseases $[8,9,10]$.

\section{Acknowledgment}

We would like to express our thanks for the support of project grant from Xiamen science and technology project (Grand No. 3502Z20144025), China.

\section{References}

[1] S.Y. Duan, S.M. Lv, F. Ye and L.B. Chen. Three-dimensional CTA study on the relations between the vertebral artery and atlantoaxial joint, Chinese medical journal. 122 (2009) 917-920.

[2] M.P. Steinmetz, T.E. Mroz and E.C. Benzel. Craniovertebral junction: biomechanical considerations, Neurosurgery. 66 (2010) 7-12.

[3] H.H.Wang, Z.B.Shen, Z.Deng, K.Wang and H.S. Zhan.Construction of a human cervical spine with bilateral vertebral artery fluid-solid coupling model, Zhejiang Da Xue Xue Bao Yi Xue Ban. 44(2015) 131-137 (In Chinese).

[4] C.W. Pfirrmann, C.A. Binkert, M. Zanetti, N. Boos and J. Hodler J. Functional MR imaging of the craniocervical junction. Correlation with alar ligaments and occipito-atlantoaxial joint morphology: a study in 50 asymptomatic subjects, Schweiz Med Wochenschr. 130 (2000) 645-651.

[5] H. Zhang and J. Bai. Development and validation of a finite element model of the occipito atlantoaxial complex under physiologic loads, Spine (Phila Pa 1976). 32 (2007) 968-974.

[6] C. Meng, S. Yang and P. Wang. Research on biomechanics properties of occipito - atlantoaxial complex by finite element method, Sheng Wu Yi Xue Gong Cheng Xue Za Zhi. 27 (2010) 1173-1177 (In Chinese).

[7] Kleinberger M. Application of finite element techniques to the study of cervical spine mechanics, Proceedings of the 37th Stapp Car Crash Conference (San Antonio, USA, Nov.7-8, 1993) Vol 1, p.261-272.

[8] S.Y. Duan, L. Zhang, C.H. Lin and H.Zhong. Biomechanical simulation of atlantoaxial joint with vertebral artery, basic \& clinical pharmacology \& toxicology. 117 (2015) 26-26.

[9] S. Patkar.Anterior facetal realignment and distraction for atlanto-axial subluxation with basilar invagination ... a technical note, Neurol Res. 17(2016) E1-3.

[10]S. Patkar. New entry point for C2 screw, in posterior C1-C2 fixation (Goel-Harm's technique) significantly reducing the possibility of vertebral artery injury, Neurol Res. 38 (2016) 93-97. 